\title{
Performance Analysis of NEMO using City Section Mobility Model
}

\author{
Md. Shohrab Hossain, Mohammed Atiquzzaman, William Ivancic $\dagger$ \\ School of Computer Science, University of Oklahoma, Norman, OK 73019 \\ $\dagger$ NASA Glenn Research Center, Cleveland, OH 44135 \\ shohrab@ou.edu, atiq@ou.edu,wivancic@grc.nasa.gov
}

\begin{abstract}
Mobile networks can be formed in bus, train, aircrafts, satellites with a wide variety of on-board IP-enable devices and Network Mobility (NEMO) protocols are required to support uninterrupted services to ongoing sessions. Node mobility has a direct impact on the performance evaluation of various NEMO protocols. However, most of the analysis on mobility protocols used random waypoint mobility model which does not always represent real-world movement patterns in city streets. In this paper, we have used city section mobility model, a realistic street mobility model, to analyze the performance of the basic network-mobility protocol. We have used $n s-2$ simulation to compare the performance of NEMO using city section and random waypoint models. Results have been obtained for average throughput, packet drop probability, end-to-end delay, handoff frequency, and signaling overhead and show significant deviation between the mobility models. Our analysis thus can help in estimating the various performance metrics of mobile network deployed in city streets.
\end{abstract}

Keywords: computer networks, mobility model, network mobility, ns-2 simulation, performance analysis.

\section{INTRODUCTION}

Hosts, and mobile routers, managing the mobility of hosts, constitute the mobile network. Mobile networks can be formed in bus, train, aircrafts with a wide variety of on-board IP-enable devices that access Internet. To efficiently manage the mobility of multiple IP-enabled hosts moving together, Internet Engineering Task Force proposed NEtwork MObility Basic Support Protocol (NEMO BSP) [1]. In NEMO, mobile router having higher transmission capability acts as the gateway for the nodes inside the network.

To mimic the movement pattern of mobile hosts and networks, mobility models are used. Mobility should be modeled in a realistic manner, otherwise simulation result may not fit well in a real world deployment. Examples of mobility models are Random Walk, Random WayPoint (RWP), Random Direction, Gauss-Markov, and City Section. Among them, RWP model is the most common and frequently used model due to its simplicity. But the movement pattern represented by RWP model is not always realistic. It picks a random speed and direction, resulting in sharp turns and sudden stops frequently which is quite unusual while moving along city streets. On the other hand, city section mobility model, introduced by Davies et al. [2], [3], represents movement behavior influenced by constraints in the environment, avoiding obstacles, buildings etc. Thus city section mobility model is a realistic movement pattern for vehicles in a city.

In our earlier attempts [4]-[6], we have evaluated the performance of various host and network mobility protocols. In [4], we have compared the handoff performance of network-mobility protocols using an experimental testbed. In [5], we have used analytical performance model and experimental testbed for comparison for network-mobility protocols with respect to transmission delay, throughput, number of binding updates. In [6], cost analysis of network mobility protocols has been performed. In all these works [4]-[6], we have used random waypoint mobility model as the underlying mobility model which cannot be used while moving in city streets. However, we have not evaluated the performance of network mobility protocols when different mobility models are used, rather the performance comparison have been carried out among various mobility protocols.

Our objective in this paper is to demonstrate quantitatively that the city section model, which is suitable model for street mobility, has far different performance results compared to the most frequently used RWP model. This paper does not aim at showing which mobility model is better.

Our contributions in this paper are (i) performance analysis of NEMO BSP through ns-2 simulation using city section mobility model by determining average throughput, average packet drop probability, handoff frequency, average end-to-end delay, and signaling overhead, (ii) comparison of the results with random waypoint mobility model, and (iii) validation of expected epoch length, number of subnet crossings, and subnet residence time of CSM model with our earlier analytical model [7].

The performance results obtained using CSM model are found to be far different from RWP model showing that RWP mobility model cannot approximate the street mobility model. In addition, this performance results of CSM model can be of special interest for public or military mobile networks deployed in city for urban warfare or other missions.

The rest of the paper is organized as follows. In section II, NEMO architecture and BSP are explained in brief. Section III describes some of the stochastic properties of two user mobility models: CSM and RWP. In Section IV, the performance analysis of NEMO BSP using ns-2 simulation is presented along with the comparison of the 


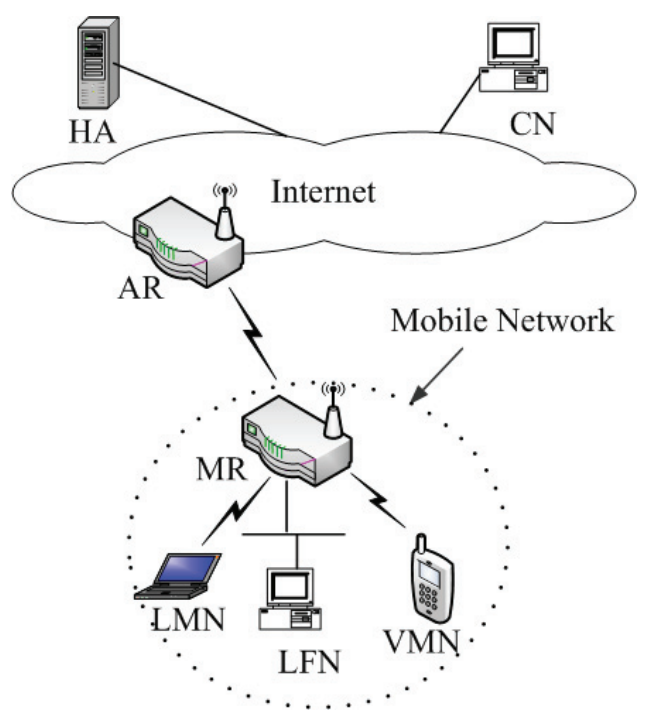

Fig. 1. Architecture of a mobile network.

results. Finally, we conclude the paper in Section V.

\section{NETWORK MOBILITY}

NEMO architecture and NEMO BSP are explained here.

\section{A. NEMO Architecture}

Fig. 1 shows the architecture of a Mobile Network (MN). Mobile Router (MR) act as gateways for the nodes inside the MN, each of the nodes are called a Mobile Network Node (MNN). Different types of MNNs are: Local Fixed Nodes (LFN) that do not move with respect to MN, Local Mobile Nodes (LMN) that usually reside in MN and can move to other networks, and Visiting Mobile Nodes (VMN) that get attached to the MN from another network. LMNs and VMNs are MIPv6 capable, and we refer them as mobile nodes. The MR attaches to the Internet through Access Routers (ARs). An MN is usually connected to a network (home network) where an MR is registered with a router called the Home Agent (HA). The HA is notified location of the MR, and redirects packets, sent by Correspondent Node $(\mathrm{CN})$ to MNNs.

\section{B. NEMO BSP}

In NEMO BSP [1], the MR ensures connectivity of all hosts inside the MN when the MR changes its point of attachment to the Internet while moving from a home network to a foreign network. An MR has its unique IP address and one or more MN Prefixes (MNP) that it advertises to the hosts attached to it. MR establishes a bidirectional tunnel with its HA to pass all the traffic between its hosts and the CNs. When MR changes its point of attachment, it acquires a new care-of-address from the visited foreign network. It then sends a Binding Update (BU) to its HA which creates a cache entry, binding MRs home address with its care-of-address, and creates a bidirectional tunnel between HA and MR. When a $\mathrm{CN}$ sends a packet to a MNN, the packet is

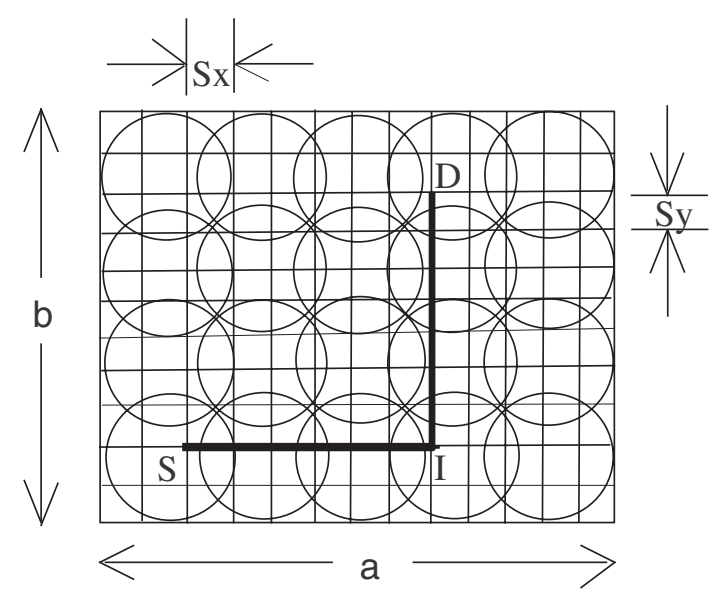

Fig. 2. Road network in city section mobility model.

routed to the HA of the corresponding MR. HA looks at its cache entry and forwards the packet to MR using a bidirectional tunnel. Finally, MR receives the packet, decapsulates it, and forwards it to the MNN.

\section{USER MOBILITY MODELS}

In this section, we explain the CSM and RWP models.

\section{A. City Section Mobility Model}

The simulation area used in CSM model [3] is represented by a grid of streets forming a particular section of a city. The model sets parameters such as speed limit of each street. Each MN begins the simulation at a predefined intersection of two streets. It then randomly chooses a destination, also represented by intersection of two streets. Moving to this destination involves (at most) one horizontal and one vertical movement. Upon reaching the destination, the $\mathrm{MN}$ pauses for some random time and then chooses another destination randomly and the process is repeated. Each such cycle is termed as an epoch. The stochastic properties of CSM model has been analyzed in [7]. The results are summarized here.

Let the environment be a rectangular area of dimension $a \times b$ as shown in Fig. 2 where roads are parallel to axes. Let there be $N_{s}$ horizontal roads (streets) and $N_{a}$ vertical roads (avenues) and streets be $S_{y}$ distance apart and avenues be $S_{x}$ distance apart. So number of streets are $N_{a}=a / S_{x}+1$, and number of avenues are, $N_{s}=$ $b / S_{y}+1$. Therefore, the expected epoch length [7] can be obtained as

$$
E(L)=\frac{a\left(N_{a}+1\right)}{3 N_{a}}+\frac{b\left(N_{s}+1\right)}{3 N_{s}}
$$

For large values of $N_{a}$ and $N_{s}, E(L)=a / 3+b / 3$.

If the speed of the mobile node vary between $V_{x}^{\text {min }}$ to $V_{x}^{\max }$ for some horizontal road segment, and between $V_{y}^{\min }$ to $V_{y}^{\max }$ for some vertical road segment, then the expected epoch time [7] is given by,

$E(T)=E\left(L_{x}\right) \frac{\ln \left(V_{x}^{\max } / V_{x}^{\min }\right)}{V_{x}^{\max }-V_{x}^{\min }}+E\left(L_{y}\right) \frac{\ln \left(V_{y}^{\max } / V_{y}^{\min }\right)}{V_{y}^{\max }-V_{y}^{\min }}$ 
where $V^{\max }>V^{\min }$

In order to avoid collisions at each road intersection, there will be a random pause time between 0 to $P_{\max }$. So the mean pause time is $E(P)=P_{\max } / 2$. The expected number of subnet crossing in an epoch [7] is :

$$
\begin{aligned}
& E(C)=\frac{m(m+1) K}{6 N_{a}^{2}}\left(6 N_{a}-4 m K+K+3\right) \\
& +\frac{n(n+1) K}{6 N_{s}^{2}}\left(6 N_{s}-4 n K+K+3\right)
\end{aligned}
$$

Therefore, average residence time in a subnet can be estimated as follows [7]:

$$
T_{r}=\frac{E(T)+2 E(P)}{E(C)}
$$

\section{B. Random Waypoint Mobility Model}

A similar analysis on RWP model is done in [8] and the expected epoch length can be obtained as [8]:

$$
\begin{aligned}
& E\left(L^{\prime}\right)=\frac{1}{15}\left[\frac{a^{3}}{b^{2}}+\frac{b^{3}}{a^{2}}+\sqrt{a^{2}+b^{2}}\left(3-\frac{a^{2}}{b^{2}}-\frac{b^{2}}{a^{2}}\right)\right] \\
& +\frac{1}{6}\left[\frac{b^{2}}{a} \Phi\left(\frac{\sqrt{a^{2}+b^{2}}}{b}\right)+\frac{a^{2}}{b} \Phi\left(\frac{\sqrt{a^{2}+b^{2}}}{a}\right)\right]
\end{aligned}
$$

where $\Phi()=.\ln \left(.+\sqrt{(.)^{2}-1}\right)$

The expected epoch time for RWP model is given by the following equation [8]:

$$
E\left(T^{\prime}\right)=E\left(L^{\prime}\right) \frac{\ln \left(V_{\max } / V_{\min }\right)}{V_{\max }-V_{\min }}
$$

The average pause time of RWP model is $E\left(P^{\prime}\right)=$ $P_{\max } / 2$. The expected number of subnet crossings per epoch for RWP model is given by the following equation [8]:

$$
E\left(C^{\prime}\right)=\frac{1}{m^{2} n^{2}} \sum_{\alpha_{j}=1}^{m} \sum_{\beta_{j}=1}^{n} \sum_{\alpha_{i}=1}^{m} \sum_{\beta_{i}=1}^{n}\left(\left|\alpha_{i}-\alpha_{j}\right|+\left|\beta_{i}-\beta_{j}\right|\right)
$$

Therefore, the subnet residence time of RWP model can be estimated as follows [8]:

$$
T_{r}^{\prime}=\frac{E\left(T^{\prime}\right)+E\left(P^{\prime}\right)}{E\left(C^{\prime}\right)}
$$

\section{PERFORMANCE EVALUATION}

For the performance evaluation of NEMO BSP, we have performed ns-2 simulation [9]. Simulation environment, analysis of the results and a comparative discussion are presented in the following subsections.

\section{A. Simulation Environment}

Fig. 3 shows the topology used for the simulation. The simulation area is covered by 100 access routers (arranged in 10 rows and 10 columns); each access point's transmission range is $250 \mathrm{~m}$. IEEE $802.11 \mathrm{~b}$ standard is used for wireless communications whereas LFNs are connected to MRs through Ethernet (IEEE 802.3). The number of LFNs in the MN is varied from 1 to 10 , channel capacity is $2 \mathrm{Mbps}$. The hierarchical addresses of all the nodes are listed in Table I and values used for parameters are listed in Table II.

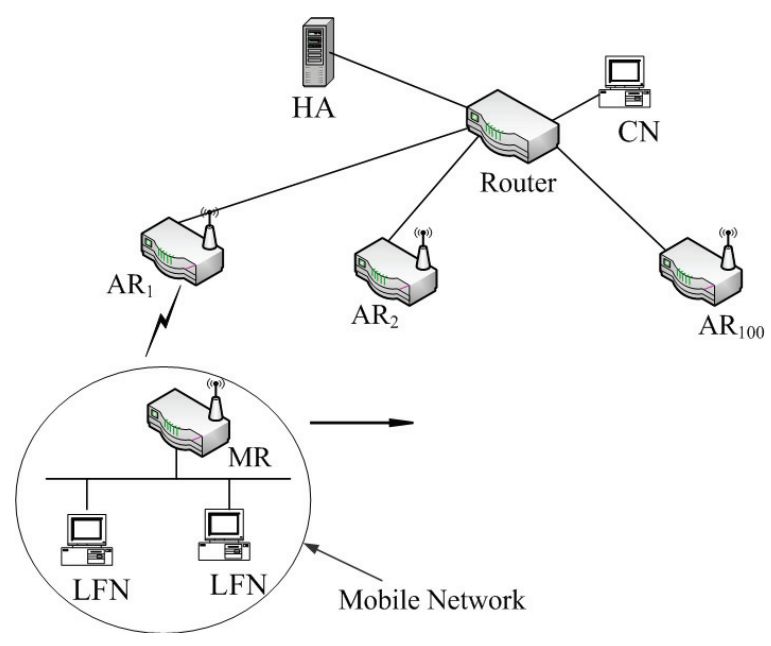

Fig. 3. Simulation Topology for NEMO

TABLE I

HIERARCHICAL ADDRESSES OF THE SIMULATION ENTITIES.

\begin{tabular}{|l|l|}
\hline Node Type & Hierarchical addresses \\
\hline CN & 0.0 .0 \\
\hline Router & 1.0 .0 \\
\hline HA & 1.1 .0 \\
\hline MR & 1.1 .1 \\
\hline Gateway (MN) & 1.1 .2 \\
\hline $\mathrm{LFN}_{1}-\mathrm{LFN}_{10}$ & $1.1 .3-1.1 .12$ \\
\hline $\mathrm{AR}_{1}-\mathrm{AR}_{100}$ & $1.1 .0-1.1 .100$ \\
\hline
\end{tabular}

$\mathrm{CN}$ is an FTP source over TCP whereas LFNs of the mobile network are the TCP sinks. Each simulation is run for $700 \mathrm{sec}$ for CSM and RWP mobility models, and in each run the MN moves in the environment covered by the 100 ARs. The simulation was run first by varying number of active LFNs in the MN (starting from 1 LFN and varying up to 10) with a random speed between 10 to $20 \mathrm{~m} / \mathrm{s}$. Next, simulation was run varying the speed of the $\mathrm{MN}$ from $5 \mathrm{~m} / \mathrm{s}$ to $35 \mathrm{~m} / \mathrm{s}$ while using 5 active LFNs. We have run each simulation 10 times and taken the average of the results.

\section{B. Results}

The results obtained from the analysis of the simulation traces are presented in the following. We have measured

TABLE II

VALUES OF PARAMETERS USED IN THE SIMULATION.

\begin{tabular}{|l|l|}
\hline Parameter & Value \\
\hline Simulation time & $700 \mathrm{~s}$ \\
\hline Wired link BW & $10 \mathrm{Mbps}$ \\
\hline Wired link delay & $1.8 \mathrm{~ms}$ \\
\hline Wireless (802.11b) link BW & $11 \mathrm{Mbps}$ \\
\hline Wireless range & $250 \mathrm{~m}$ \\
\hline Ethernet (802.3) BW & $10 \mathrm{Mbps}$ \\
\hline Simulation area & $3686 \times 3686 \mathrm{~m}^{2}$ \\
\hline Inter-road spacings & $150 \mathrm{~m}$ \\
\hline Number of Access Points & 100 \\
\hline Number of LFNs in MN & $1-10$ \\
\hline Speed of MN & $5-35 \mathrm{~m} / \mathrm{s}$ \\
\hline
\end{tabular}


average throughput, average packet drop probability, handoff frequency, average end-to-end delay, number of binding updates.

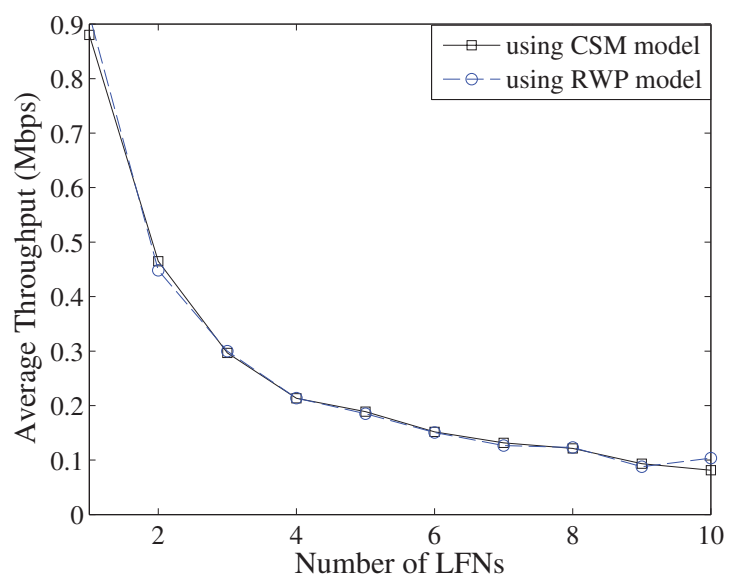

(a)

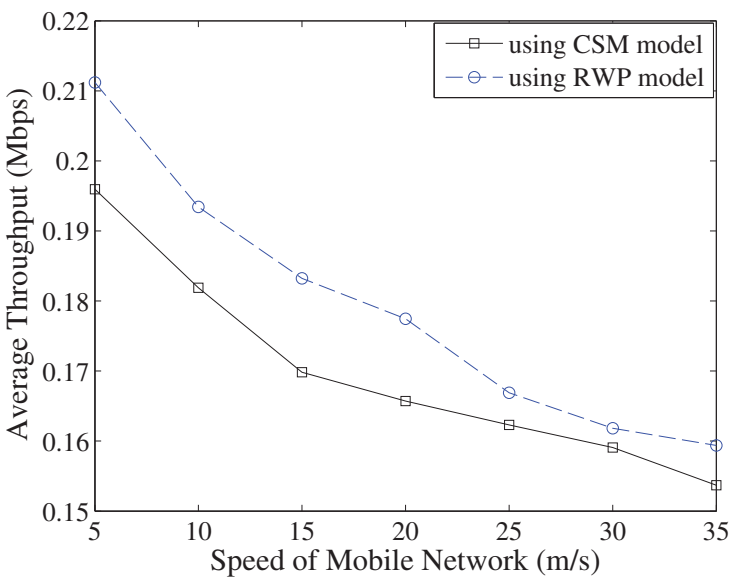

(b)

Fig. 4. Average throughput vs. (a) number of LFNs and (b) speed of MN using CSM and RWP models.

1) Average Throughput: In Fig. 4(a), average throughput at $\mathrm{LFN}_{1}$ is shown for varying number of active LFNs in the MN using CSM and RWP model. As number of LFNs communicating increases, the throughput decreases due to contention for the wireless media. The average values are close but there are an average of $5 \%$ variation between these two models. This is because of the variation of movement patterns between the two mobility models.

In Fig. 4(b), average throughput at $\mathrm{LFN}_{1}$ is shown for varying speeds of the MN. We use 5 LFNs in the MN. Average throughput decreases for higher speeds due to increase of handoff frequency, resulting in higher packet drop. Average throughput are found to be much different for two mobility models with a variation of 1.7 to $8 \%$. This is due to the differences in epoch lengths and epoch times of the two models.

2) Average Packet Drop Probability: To compute the packet drop probability, we have counted the number of packets sent by $\mathrm{CN}$, and number of packets received by the LFN. Thus packet drop probability is the ratio

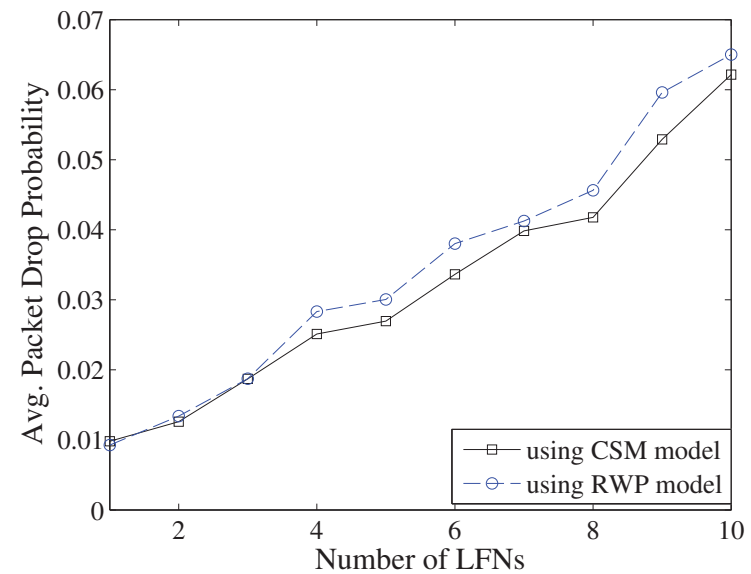

(a)

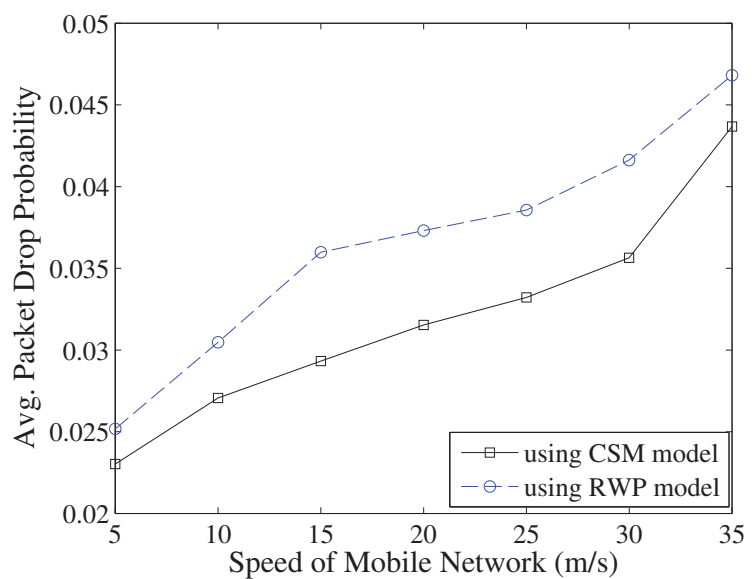

(b)

Fig. 5. Average packet drop probability vs. (a) number of LFNs and (b) speed of MN using CSM and RWP models.

of dropped packets to the total packets sent by $\mathrm{CN}$. In Fig. 5(a), average packet drop probability is shown for varying number of active LFNs using CSM and RWP model. The packet drop probability increases for higher number of LFNs in the MN due to contention for the media. The differences of drop probability for CSM and RWP models are found to be between 0 to $13 \%$.

In Fig. 5(b), average packet drop probability is shown for varying speed of the MN. Average drop probability increases for higher speed of MN due to higher number of handoff. It is also found that two mobility models have significantly different (7-22\%) packet drop probability.

3) Handoff frequency: In Fig. 6(a) shows number of handoffs for varying number of active LFNs in the MN using CSM and RWP model. The average handoff frequencies for CSM and RWP models are found to be 35 and 44 , respectively. The variation of handoff frequencies between the mobility models are due to their movement patterns. In Fig. 6(b) shows number of handoffs for varying speed of the mobile network using CSM and RWP model. They are also found to be significantly different (2-18\%) for two mobility models.

4) Average end-to-end delay: End-to-end delay is measured by the difference between the time of sending 


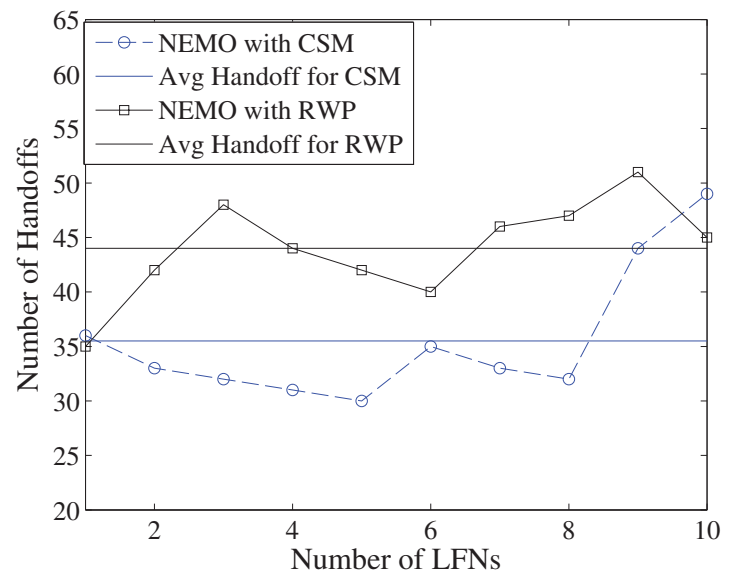

(a)

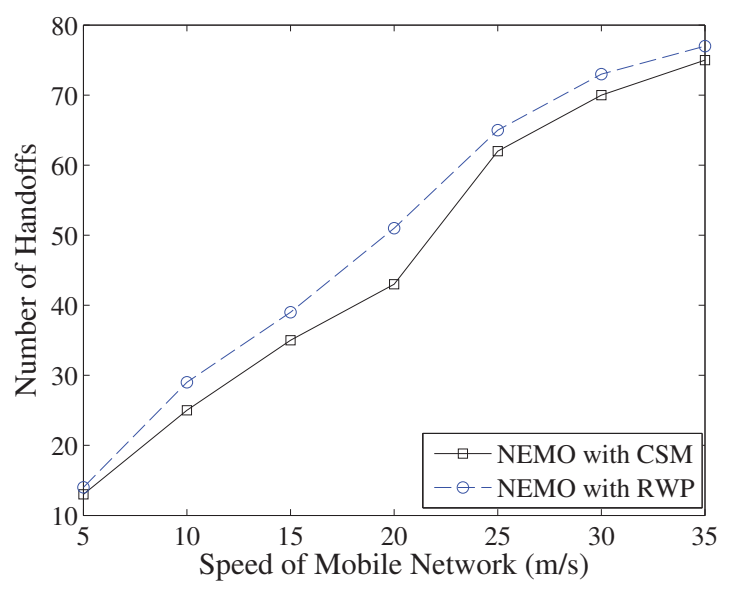

(b)

Fig. 6. Handoff frequency vs. (a) number of LFNs and (b) speed of MN using CSM and RWP models.

the packet by $\mathrm{CN}$ and time of receiving the packet by LFN. We have measured the delay for for each of the data packets sent by $\mathrm{CN}$, and took average of those. In Fig. 7(a), average delay is shown for varying number of active LFNs in the MN using CSM and RWP model. Average delay increases for higher number of LFNs due to queuing delay and contention of the media. The variation between the delays of CSM and RWP models are between 4.8 to $22.2 \%$. In Fig. 7(b), average packet delay is shown for varying speed of mobile network using CSM and RWP model and their averages are 0.217 and $0.207 \mathrm{sec}$ with a deviation of $4.6 \%$.

5) Binding update messages: The simulation was run for several speeds of MN, starting from $5 \mathrm{~m} / \mathrm{s}$ to $35 \mathrm{~m} / \mathrm{s}$. We have counted the number of binding acknowledgement received by the MR in the total simulation time as shown in Fig. 8. Number of binding updates increases with higher MN mobility. It is found that the movement pattern of RWP model causes more Binding updates to be sent by the MR due to higher number of handoff in RWP compared to that of CSM which is also evident from Fig. 6(b).

6) Expected Epoch Length: According to the theoretical model [7] of CSM model, the expected epoch length

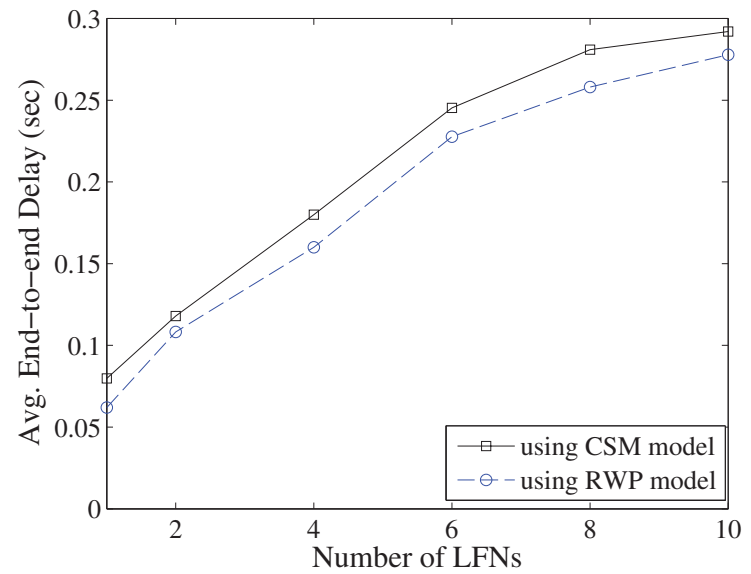

(a)

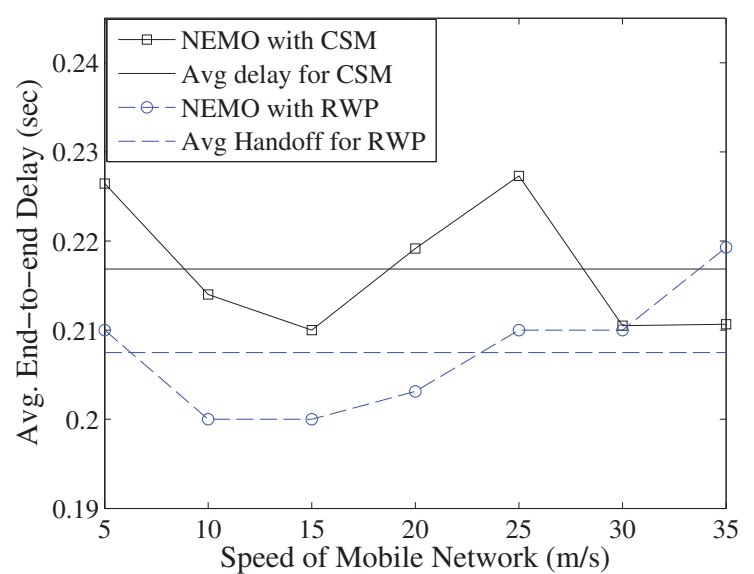

(b)

Fig. 7. Average end-to-end delay vs. (a) number of LFNs and (b) speed of MN using CSM and RWP models.

of the environment is given by Eqn. (1). Hence, $E(L)=$ $E\left(L_{x}\right)+E\left(L_{y}\right)=2 *(3686 / 3)=2457.3 m$ Using the simulation, we found that total distance covered in 50 epochs is 121590 . Hence, the mean epoch length is $=121590 / 50=2431.8 \mathrm{~m}$ which are close to the theoretical value.

7) Expected number of subnet crossings: For, computing the number of subnet crossing in CSM model, we need the values of $N_{a}$ and $N_{s}$ (see section IIIA). $N a=3686 / 150+1=26=N_{s}$. And also $K=\lfloor 2 r / S x\rfloor=\lfloor(2 \times 250 / 150)\rfloor=3$. Using the Eqn. (3), we find that $E(C)=6.8$. From the ns-2 simulation traces, the average number of subnet crossing per epoch was found to be 6.72 .

8) Subnet Residence Time: If the mobile network moves in the environment between the speed $14 \mathrm{~m} / \mathrm{s}$ to $24 \mathrm{~m} / \mathrm{s}$, the expected epoch time in CSM model can be computed by Eqn. (2). Since the grid area is squareshaped, $E\left(L_{x}\right)=E\left(L_{y}\right)=E(L) / 2=1228.65 \mathrm{~m}$. Thus $E(T)=2 \times 1228.65 \times \ln (24 / 14) / 10=132.44 \mathrm{sec}$. As maximum pause time is $2 \mathrm{sec}$, the subnet residence time of CSM model is $T_{r}=(132.44+2 / 1) / 6.8=19.62 \mathrm{sec}$. On the other hand, the total simulation time was 700 sec, and there were 35 handoffs in CSM model. So the 


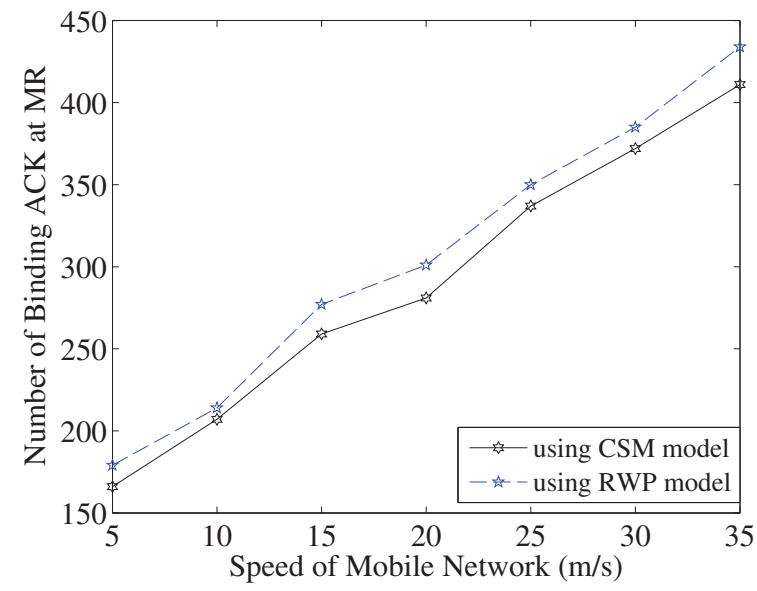

Fig. 8. Number of binding acknowledgement received at the MR for different speeds of MN using CSM and RWP models.

TABLE III

DIFFERENCES BETWEEN RESULTS OF CSM AND RWP MODELS.

\begin{tabular}{|l|l|l|}
\hline Criteria & Diff.(\%) & $\begin{array}{l}\text { Avg. } \\
\text { Diff.(\%) }\end{array}$ \\
\hline Avg. throughput vs. number of LFNs & $0.1-27$ & 5 \\
\hline Avg. throughput vs. speed of MN & $1.7-8$ & 5.3 \\
\hline Avg. pkt. drop prob. vs. number of LFNs & $0-13$ & 7.9 \\
\hline Avg. pkt. drop prob. vs. speed of MN & $7-22$ & 14.7 \\
\hline Number of handoff vs. number of LFNs & $2.7-50$ & 28 \\
\hline Number of handoff vs. speed of MN & $2-18$ & 9.3 \\
\hline Avg. end-to-end delay vs. number of LFNs & $4.8-22$ & 10.3 \\
\hline Avg. end-to-end delay vs. speed of MN & $0.2-7.6$ & 4.6 \\
\hline Number of BU messages vs. speed of MN & $3.3-7.8$ & 5.4 \\
\hline
\end{tabular}

average subnet residence time was found to be $20 \mathrm{sec}$.

\section{A. Comparative Discussion}

For a comparative analysis, the percentage difference and the mean percentage difference between the results of CSM and RWP model are listed in Table III. It is evident that the performance metrics of RWP model differ significantly with that of CSM model. The percentage difference is between $4.6 \%$ and $28 \%$. In RWP model, the movement pattern unrestricted and the $\mathrm{MN}$ is allowed to move along any direction whereas that of CSM model is rather restricted by the environment and the speed limits. The handoff frequencies of the mobility models varies between 2 to $18 \%$ with respect to various speed of the mobile network. This difference in handoff frequency affects the throughput, average endto-end delay and packet drop probability. In short, the performance metrics of NEMO BSP varies significantly for two mobility models.

In Table IV, we have listed the results of the stochastic properties of CSM model obtained using the mathemat- ical model [7] and by the simulation. The theoretical values of mean epoch length, mean number of subnet crossing and subnet crossing times matches that obtained by simulation, thus validating the model [7].

\section{CONCLUSION}

In this paper, we have analyzed the performance of NEMO BSP using city section mobility model, a realistic street mobility model through ns-2 simulation. We have obtained results for the average throughput, average packet drop probability, handoff frequency, end-to-end packet delay, and signaling overhead using city section and random waypoint model. The performance metrics of random waypoint model differ significantly with that of city section model due to the variation in movement pattern. Hence, random waypoint model cannot be used as an approximation to a realistic street mobility like city section mobility model. Our analysis presented in this paper can help in estimating the various performance metrics of mobile network-equipped with mobile routers that can connect to wireless access points-deployed in city streets. This will facilitate Internet connectivity to vehicles with IP-enable devices, ensuring short and long range real-time communication.

\section{ACKNOWLEDGEMENT}

The research reported in this paper was funded by NASA Grant NNX06AE44G.

\section{REFERENCES}

[1] V. Devarapalli, R. Wakikawa, A. Petrescu, and P. Thubert, "NEtwork MObility (NEMO) basic support protocol," RFC 3963, Jan 2005 .

[2] T. Camp, J. Boleng, and V. Davies, "A survey of mobility models for ad hoc network research," Wireless Communications and Mobile Computing, vol. 2, pp. 483-502, 2002.

[3] V. Davies, "Evaluating mobility models within an Ad Hoc Network," MS thesis, Colorado School of Mines, 2000.

[4] M. S. Rahman, O. Bouidel, M. Atiquzzaman, and W. Ivancic, "Performance Comparison between NEMO BSP and SINEMO," in IEEE GLOBECOM, New Orleans, LA, Nov 30- Dec 42008.

[5] P. Chowdhury, A. Reaz, M. Atiquzzaman, and W. Ivancic, "Performance analysis of SINEMO: Seamless IP-diversity based Network Mobility," in ICC 2007, Glasgow, Scotland, June 24-28, 2007.

[6] A. S. Reaz, P. K. Chowdhury, and M. Atiquzzaman, "Signaling cost analysis of SINEMO: Seamless End-to-End Network Mobility," in First ACM/IEEE International Workshop on Mobility in the Evolving Internet Architecture, San Francisco, CA, Dec 01, 2006.

[7] M. S. Hossain and M. Atiquzzaman, "Stochastic properties and application of city section mobility model," in IEEE Global Communications Conference (GLOBECOM), Honolulu, HI, Nov 30- Dec 4, 2009.

[8] C. Bettstetter, H. Hartenstein, and X. Prez-Costa, "Stochastic properties of Random Waypoint mobility model," Wireless Networks, vol. 10, no. 5, pp. 555-567, Sept. 2004.

[9] "The network simulation - ns-2," http://www.isi.edu/nsnam/ns/.
TABLE IV

ANALYTICAL AND SIMULATION RESULTS OF CSM MODEL.

\begin{tabular}{|l|l|l|l|}
\hline Type of Results & $\mathbf{E}(\mathbf{L})$ & $\mathbf{E}(\mathbf{C})$ & $\mathbf{T}_{r}$ \\
\hline Analytical & $2457.3 \mathrm{~m}$ & 6.8 & $19.62 \mathrm{sec}$ \\
\hline Simulation & $2431.8 \mathrm{~m}$ & 6.72 & $20 \mathrm{sec}$ \\
\hline
\end{tabular}

